\title{
The failure of "engineered rejuvenation": laboratory genomics do not translate into precision anti-aging therapies
}

\author{
Marios Kyriazis \\ ELPIs Foundation for Indefinite Lifespans, Larnaca 6021, Cyprus. \\ Correspondence to: Dr. Marios Kyriazis, ELPIs Foundation for Indefinite Lifespans, Larnaca 6021, Cyprus. E-mail: drmarios@live.it
}

How to cite this article: Kyriazis M. The failure of "engineered rejuvenation": laboratory genomics do not translate into precision antiaging therapies. J Trans/Genet Genom 2018;2:7. https://doi.org/10.20517/jtgg.2018.04

Received: 2 Apr 2018 First Decision: 7 May 2018 Revised: 21 May 2018 Accepted: 22 May 2018 Published: 15 Jun 2018

Science Editor: Sheng-Ying Qin Copy Editor: Jun-Yao Li Production Editor: Huan-Liang Wu

\begin{abstract}
There is a general failure of reductionist and mechanistic approaches to rejuvenation biomedical technologies which aim at providing treatments against aging (defined as "time-dependent dysfunction"). Importantly, it is becoming increasingly recognised that genomic research findings in animals may not adequately be translated into effective human anti-aging therapies. There exist translational impediments, which although individually formidable, can theoretically be overcome. However, the combined effects of these obstacles render this reductionist avenue of quest unattainable, at least for the foreseeable future. Some of the clinical problems of physical and genomic-based therapies against aging include side effects, interactions, inter-subject variability, compliance, patient self-reporting of data, motivation, administrative issues, infrastructure, etc. A systematic review spanning over the past 5 years, describes these problems and identifies novel approaches. New and emerging disciplines and concepts such as molecular pathological epidemiology, social genomics and other "systems-thinking" methods provide a more comprehensive view of the entire subject of aging, and study its indivisible bonds with the environment, society and culture. The so-called "fountain of youth" cannot be found in a physical item.
\end{abstract}

Keywords: Reductionism, rejuvenation biotechnologies, genomic research, translation, systems-thinking, molecular pathological epidemiology

\section{INTRODUCTION}

Research into the aging process has flourished over the past several decades, and has provided a generally informative glimpse into the intricacies of aging mechanisms ${ }^{[1,2]}$. Reductionist approaches (i.e., analyzing the mechanisms of aging in terms of its simple constituents) have assisted in this respect, by providing

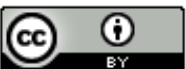

(C) The Author(s) 2018. Open Access This article is licensed under a Creative Commons Attribution 4.0 International License (https://creativecommons.org/licenses/by/4.0/), which permits unrestricted use, sharing, adaptation, distribution and reproduction in any medium or format, for any purpose, even commercially, as long as you give appropriate credit to the original author(s) and the source, provide a link to the Creative Commons license, and indicate if changes were made.

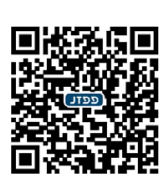


a framework explaining certain basic biological mechanisms. In a reductionist model, we consider a complex issue such as aging, and then choose to study in detail a central, albeit limited component ${ }^{[3]}$. Unfortunately, this has also encouraged others to develop a more widespread reductionist thinking in terms of therapeutic approaches, and examples abound in the popular literature (see, for example: https:// www.calicolabs.com, and https://en.wikipedia.org/wiki/Strategies_for_Engineered_Negligible_Senescence). Reductionism works up to a point, but then a more comprehensive "systems thinking" has to be developed with regards to treating aging ${ }^{[4,5]}$. This explores the complex relationships between the parts of a dynamic system in relation to the whole, and the interdependency of such structures of dynamic systems ${ }^{[6]}$. Despite a large number of reports that a drug, nutrient, procedure, compound or other, prolongs healthy lifespan in laboratory animals, human beneficial mirroring effects have yet to be realized ${ }^{[7-17]}$. In part, this is due to the reductionist method employed by the vast majority of researchers which does not take into account the unique characteristics of humans. This review paper concentrates on evidences pointing at the difficulties that may be encountered during such reductionism-based rejuvenation approaches. Here, rejuvenation essentially means "the reversal of the biological process of aging". The paper also concentrates on the specific cases of genetic therapies and presents evidences about the difficulties that may be encountered in developing effective medical rejuvenation therapies and in translating laboratory genomic research into everyday clinical practice.

The review is based on systematic literature search of the databases of PubMed, Embase, Google Scholar, Medline and Cochrane, concentrating mainly on papers published in the last 5 years. Papers presenting a "systems thinking" approach are considered, and the main concepts are included, strengthening the general thrust of this review. Papers presenting researches based on reductionism are also included, in order to highlight the contrast between the current approach and a more complex-oriented thinking. The purpose is to concentrate on emerging thinking pointing at a more systematic consideration of aging, instead of merely repeating well-known reductionist and mechanistic themes.

\section{DISCUSSION}

In the world of scientific research aiming to provide treatments for age-related disorders, it is becoming increasingly relevant to think along the mantra of our times: "To provide the right care to the right patient at the right time". Nevertheless, some researchers and academics ${ }^{[18]}$ study the scope of disruptive biotechnologies and other reductionist models, hoping that this approach may help repair specific agerelated damage, reduce the impact of degeneration and thus increase healthy lifespan. The translational problems, however, remain formidable ${ }^{[19-22]}$. The difficulties in translating laboratory anti-aging research into clinical practice are very easily appreciated by a simple test. A PubMed online search (18 May 2018) of the term "rejuvenation biotechnologies in aging" shows 99 papers discussing theoretical or laboratory aspects of rejuvenation biotechnologies. Instead, a search of "clinical applications of rejuvenation biotechnologies in aging", reveals just one relevant paper ${ }^{[23]}$. Henney ${ }^{[22]}$ states: "Putting it bluntly, how can such reductionist approaches possibly help us predict and test, let alone understand how a new medicine will work, when given to a patient in the target treatment group, which, in the case of the major illnesses that are being targeted by personalization strategies, is likely to be over 65 , with a number of co-morbidities and already taking a number of other medications? So is it really that surprising to encounter failures in efficacy in the clinic? In our understandable enthusiasm to follow the path laid by the genome revolution, generating an evermore intricate map of human disease at the molecular level, we appear to have forgotten, or at least relegated the importance of an understanding of the physiological context".

Instead of considering general approaches, it is imperative to focus on concepts derived from an emerging branch of medicine, namely precision medicine $(\mathrm{PM})^{[24]}$. In $\mathrm{PM}$, healthcare in general, and therapies in particular, are customized to the needs of individual patients and can be based on the patient's specific genetic 
profile. This may render more effective therapies with fewer side effects. PM may also include the creation of unique combinations of treatments that may benefit specific patients. Based on this model of medicine, we immediately encounter a problematic contrast with current rejuvenation research. Such research aims to discover therapies that may be effective in anti-aging, as well as therapies that will be common to all, ignoring the fact that the aging patient is a unique individual with unique genetic profile and environment. Instead, a PM-centered approach is needed here ${ }^{[22,25]}$. This approach must be based on concepts developed in systems biology (the computational and mathematical modeling of complex biological systems and interactions) which sees the human body in a more holistic manner, in contrast to the current reductionist model. In addition, it is possible to gain insights from social genomics, i.e., how our genome is affected by external social factors ${ }^{[26]}$. The influence of these external factors on our biology are paramount. For instance, the combined effects of lifestyle elements, living environment, and personal habits are magnified when we also consider the additional influence of the genome, biological processes, clinical condition, medical history, existing diseases and medication of the target patient. The ideal goal of precision medicine is to find effective ways to prevent the development of an illness, and also provide solutions that can be helpful in the treatment of existing conditions.

It is necessary to clarify here that this paper refers to discrete physical therapies or interventions which may, perhaps, originate from genomic research, and follow a basic principle: the therapy is given by a "therapist" (such as a physician) to a "patient", in order to effect a cure of aging. Examples include not only rejuvenation biotechnologies in general, but also anti-senescence drugs (senolytics) ${ }^{[27,28]}$ such as quercetin, dasatinib, navitoclax ${ }^{[29]}$, drugs or compounds such as sirtuin modulators ${ }^{[30]}$, rapamycin ${ }^{[31]}$, lifespan-extending herbs ${ }^{[32]}$, $\operatorname{metformin}^{[33]}$, telomere and telomerase modulators ${ }^{[34]}$. A common characteristic of these is that there is a clear separation between an outside agent (a physician, surgeon, healer, nutritionist) and the patient (healthy or unhealthy young or older people).

Some translational problems associated with such biomedical technologies ${ }^{[35]}$ include practical issues with harvesting autologous stem cells or genetic material, transplantation surgery (of such material), immunosuppression, infrastructure of delivery, unknown and unpredictable side effects, reluctance to accept these technologies as a treatment, inadequate integration and assimilation of genes, unknown variables relating to genetic cross-talk and over-expression, clinical polypharmacy, drug interactions and noncompliance $^{[36]}$.

The consideration of each of these issues separately, leads to significant translational problems. However, if we also consider that these therapies must be deployed in combination in order to effect a lasting clinical benefit, it may be concluded that additional emergent problems will be manifest, and these may negate any practical clinical applications ${ }^{[37]}$. This kind of approach involving a physical therapy, an item, an intervention that exists materially and can be given physically by a separate observer (physician, healer) to the detached patient is inherently reductionist and naïve ${ }^{[38]}$. This methodology has hitherto been inadequate with regards to substantially reducing the impact of aging in human subjects ${ }^{[20,21,39]}$.

\section{ONE EXAMPLE: NON-ADHERENCE}

A typical and easy to appreciate example of where genomics fail to be adequately translated into a clinical benefit, is the issue of non-adherence to medication ${ }^{[40]}$. Perhaps, a better term would be "badadherence" meaning that the patient may indeed take the medication, but take it erratically, irregularly and inappropriately, something that will be reflected in the increased likelihood of side-effects. In this respect, there are several reasons why an effective laboratory genomic discovery may not be translated as an effective clinical therapy. Reasons include cognitive impairment and depression ${ }^{[41]}$, asymptomatic disease ${ }^{[42]}$, side effects of the medication, which in the case of aging are likely to be several because a wide range of therapeutic interventions will have to be deployed ${ }^{[43]}$, complexity of the therapeutic approach ${ }^{[44,45]}$ and even 
administrative issues (including inadequate follow-up or discharge planning, poor relationship between patient and provider, missed appointments, lack of health insurance, and cost $)^{[46]}$. Each of these reasons in isolation, may not be sufficiently considered as a significant obstacle. However, these must be considered as a whole, because in real life many such issues may affect any one specific patient. Therefore, these problems significantly reduce the likelihood that a putative anti-aging therapy can be used appropriately by any target patient, even in the case of a putative therapy based on genomic research.

\section{SOCIAL GENOMICS}

If we reject reductionism as a model against aging, where does this leave us? In this case, a more complexoriented thinking may provide some direction for future research. We know that social factors and cultural events affect the activity of our genes ${ }^{[4]}$. These interactions are studied by the emerging science of social genomics which tries to clarify the effect of socially-originating challenges, information or stimuli that may have on our biology (for example, increased expression of hundreds of gene transcripts). Cole ${ }^{[48]}$ states $^{4}$ that: "Systems-level capabilities emerge from groups of individual, socially sensitive genomes... and ... transcriptional biofeedback (that) empirically optimizes individual well-being in the context of the unique genetic, geographic, historical, developmental, and social contexts... Studies of human social genomics are now clarifying which specific types of human genes are subject to social regulation and mapping the social signal transduction pathways that mediate these effects. The results of these analyses are shedding new light on the molecular basis for social influences on individual heath, the genomic basis for human thriving, and the metagenomic capabilities that emerge from networked communities of socially sensitive genomes".

In addition, a more relevant and complex view of aging can be developed when we consider the concept of gene-culture coevolution, a concept which is associated with the general concept of social genomics ${ }^{[49]}$. The gene-culture coevolution concept explains the changing interactions between genetic evolution and cultural evolution. This is also sometimes known as dual inheritance theory ${ }^{[50]}$. An extension of this concept describes the effects from our increasingly technological culture upon our own biology ${ }^{[51]}$, and at the same time, it describes how humanity can influence the further evolution of the environment itself ${ }^{[52]}$. In this way it may be possible to appreciate the complex and mutually influencing interactions of each patient with the environment, and begin to form a view which is at odds with the simplistic reductionist view that "one drug fits all" paradigm ${ }^{[53]}$.

Furthermore, the science of social genomics and that of molecular pathological epidemiology described below explain many of the difficulties encountered in translating genomic research into clinical therapies. Therefore, we identify that the general reliance on the model which sees no connection between the physicians and the patients, is changing into a model deeming the physician, the patient and their environment to be interactive.

\section{MOLECULAR PATHOLOGICAL EPIDEMIOLOGY}

Molecular pathological epidemiology (MPE) is an emerging discipline developed, on the whole, by Harvardbased epidemiologist Ogino et al. ${ }^{[54]}$. It combines epidemiology and pathology in order to study more closely the heterogeneity and aetiology of disease, including age-related disease. The approach focuses on studies simultaneously at a microscopic (molecular, genomic, cellular), and macroscopic (organismal and population) level. MPE helps to develop a more inclusive view of the diverse processes that underlie disease progression. It is important to emphasise that concepts derived from MPE assist in a better appreciation of the influence of the environment upon genetic factors. This influence may underlie several inherently heterogeneous interacting pathological processes which may lead to disease. This makes it very difficult, if not impossible, to adequately predict, or even study, these multiple processes which can cause significant variations of diseases even in similar patients. 
Here, concepts like precision medicine (which considers the variability of individual genetic profiles in relation to the environment and lifestyle), as already mentioned, and the "unique disease principle"[55] become relevant. Essentially these affirm that each individual patient exhibit unique disease profiles based on an interaction of genetic, epigenetic and other factors such as cell interactions, nutrition, lifestyle, microbial exposure, social and racial elements, age differences etc. It is therefore essential, if we want the therapy to be successful, to develop tailor-made interventions for each patient, and not rely on generic treatments. This may prove to be an impossible task.

\section{TRANSLATION}

Traditionally, with regards to the translation of laboratory research into clinical treatments, there has been excessive reliance on a simplistic model examining the issues relating to "bench to bedside" principle. However, taking a more "systems thinking" approach, reveals that there are other translational aspects which we need to take into account. For instance, there are six translational stages of an overlapping continuum that must be considered ${ }^{[56]}$ :

To - Identification of opportunities, genomic discoveries, biomarkers and basic research;

T1 - The classic "bench to bedside" model, which bridges basic research in the laboratory to possible health applications. Here, laboratory evidence is transferred into the development of new procedures for therapy or prevention, and proceeds to human testing;

T2 - The evaluation of clinical usefulness of genomic or other therapeutic applications (i.e., if the particular product or application performs better than existing therapies in everyday clinical situations);

T3 - The wider integration of genomics or other applications into routine, community based practice (i.e., for the public at large, through delivery and dissemination practices), with the purpose of ensuring that the intervention or application reaches populations whose health can benefit;

$\mathrm{T} 4$ - The overall health impact of the particular application at a population level (if the use of the application has indeed resulted in any appreciable health benefit);

T5 - In the case of aging, which is a continual process, we may also consider a T5-stage, whereby the continual effectiveness of the application at a population level remains consistently beneficial even after the passage of long periods of time.

For example, it has been suggested that less than $2 \%$ of genomics research funded by the National Cancer Institute and less than $0.5 \%$ of published cancer genomics research is beyond stage $\mathrm{T}_{1}{ }^{[56]}$. This is also the case with rejuvenation biotechnologies and anti-aging medicines which have not yet passed stage T1. Basically, it is not a matter of making a discovery, or devising a treatment/therapy or application, but it is also a matter of that application making a clear health impact at a population level. All of the above stages need to be addressed, otherwise the promise of an effective treatment remains unfulfilled.

This is not to say that the obstacles mentioned here are insurmountable by themselves, however the totality of these make the effectiveness of any putative antiaging/rejuvenation intervention, less promising and certainly less imminent.

Treating both the diseases of ageing and age-related degeneration (age-related damage at a preclinical, preventive stage) is not merely subject to biomedical interventions aiming at "fixing" genetic and cellular damage, but also depends on behavioural ones, as well as others such as cognitive factors, psychological elements and environmental influences. In order to improve health outcomes all of these factors need to be taken into account ${ }^{[57]}$. Factors which are specific to the individual may make a huge difference between significant therapeutic benefit and little or no benefit ${ }^{[58]}$.

The rise of personalised medicine, in association with elements from MPE described above, is useful, but 
in order to provide positive results this needs to be incorporated into practice, whereas, currently, most efforts are centred round directly translating animal research into human treatments, and have failed ${ }^{[59]}$. Professionals in social sciences and behavioural medicine need to be working along biomedical technicians ${ }^{[60]}$ and this is something that is not happening at present, with regards to aging.

An issue worthy of consideration, is the situation whereby a patient may just decline to undergo genetic testing in order to establish genomic status via genomic sequencing technologies. It was found that the decline rate for undergoing such studies may range from $12 \%$ to $64 \%{ }^{[61]}$. This depended on age and type of disease as well as on administrative logistics (i.e., how easy was for someone to access a clinic and enroll, privacy issues), and psychological barriers. Therefore, even if a genetic therapy was made available in principle, this does not mean that the patient will necessarily agree to receive it.

Another possible obstacle to the deliverability of genetic testing and genetic therapy is ethical considerations. Wolf et al ${ }^{[62]}$ have identified 15 different ethical areas that needed to be considered before a genetic therapy can be applied. These range from ethics of human subjects research and the ethics of biobanks and data archives, ethics of human subjects research, the ethics of professional clinical care, and ethics relating to organization, public health, outcome assessments and disparities among others. Therefore, this adds another layer of obstacles needed to be overcome before a genetic therapy against age-related damage can be applied to the public at large.

\section{CONCLUSION}

This review has highlighted evidence pointing at a situation whereby any physical therapy against aging is essentially not forthcoming. Such physical therapies may include medication, therapies based on genomics, interventions (such as stem cell manipulation) and other rejuvenation biotechnologies. An inescapable conclusion is that we need to re-think the bulk of our efforts which are based on mechanistic reductionism (the "one-tablet-fits-all" paradigm) and, instead, escalate onto a more "systems thinking-oriented" approach. Such an approach must take into account the environment and its influence on our genome, the inter-subject variability of the public at large, and translational and ethical obstacles. We need to consider more carefully new and emerging principles, such as that of precision medicine, and the unique disease principle, as well as emerging disciplines such as social genomics and MPE. The scientific quest for eliminating the impact of aging must continue, but not along the lines of the existing paradigm.

\section{DECLARATIONS}

\section{Authors' contributions}

The author contributed solely to the paper.

\section{Availability of data and materials}

Not applicable.

\section{Financial support and sponsorship}

None.

\section{Conflicts of interest}

There are no conflicts of interest with an institution or product that is mentioned and no conflicts of interest with products that compete with those mentioned in the manuscript.

\section{Ethical approval and consent to participate}

Not applicable. 


\section{Consent for publication}

Not applicable.

\section{Copyright}

(c) The Author(s) 2018.

\section{REFERENCES}

1. Liu JP. Molecular mechanisms of ageing and related diseases. Clin Exp Pharmacol Physiol 2014;41:445-58.

2. Goldsmith TC. Evolution of aging theories: why modern programmed aging concepts are transforming medical research. Biochemistry (Mosc) 2016;81:1406-12.

3. Vaiserman AM. Aging-modulating treatments: from reductionism to a system-oriented perspective. Front Genet 2014;5:446.

4. Kyriazis M. Third phase science: defining a novel model of research into human ageing. Front Biosci (Landmark Ed) 2017;22:982-90.

5. Kyriazis M. Editorial: Re-thinking ageing: a cross-disciplinary perspective (a new era for ageing). Mech Ageing Dev 2017;163:1.

6. Cabrera D, Cabrera L. Systems thinking made simple: new hope for solving wicked problems. Ithaca, NY: Odyssean; 2015.

7. Asensio-Sánchez VM. Dracula was right: the elixir of youth could be the young blood. Arch Soc Esp Oftalmol 2015;90:453.

8. Elghblawi E. Platelet-rich plasma, the ultimate secret for youthful skin elixir and hair growth triggering. J Cosmet Dermatol 2018;17:42330.

9. Girgis CM. Vitamin D and muscle function in the elderly: the elixir of youth? Curr Opin Clin Nutr Metab Care 2014;17:546-50.

10. Andersen RE, Lim DA. An ingredient for the elixir of youth. Cell Res 2014;24:1381-2.

11. Check Hayden E. Anti-ageing pill pushed as bona fide drug. Nature 2015;522:265-6.

12. Bhullar KS, Hubbard BP. Lifespan and healthspan extension by resveratrol. Biochim Biophys Acta 2015;1852:1209-18.

13. Kumar S, Lombard DB. Finding Ponce de Leon's Pill: challenges in screening for anti-aging molecules. F1000Res 2016;5:F1000 Faculty Rev-406.

14. Cottin Y, Rochette L. A member of the TGF- $\beta$ superfamily, GDF11: functions in the cardiac regeneration, perhaps an "elixir of youth?". Arch Cardiovasc Dis 2015;108:409-11.

15. Lucke JC, Diedrichs PC, Partridge B, Hall WD. Anticipating the anti-ageing pill. Lessons from the history of the oral contraceptive pill and hormone replacement therapy. EMBO Rep 2009;10:108-13.

16. Wagner G. Towards a life prolonging pill? Small molecules with anti-ageing properties. Curr Drug Targets 2006;7:1531-7.

17. Blagosklonny MV. An anti-aging drug today: from senescence-promoting genes to anti-aging pill. Drug Discov Today 2007;12:218-24.

18. Zealley B, de Grey ADNJ. Commentary on some recent theses relevant to combating aging: February 2018. Rejuvenation Res 2018;21:706.

19. Kyriazis M, Apostolides A. The fallacy of the longevity elixir: negligible senescence may be achieved, but not by using something physical. Curr Aging Sci 2015;8:227-34.

20. Kyriazis M. The impracticality of biomedical rejuvenation therapies: translational and pharmacological barriers. Rejuvenation Res 2014;17:390-6.

21. Callahan CM, Foroud T, Saykin AJ, Shekhar A, Hendrie HC. Translational research on aging: clinical epidemiology as a bridge between the sciences. Transl Res 2014;163:439-45.

22. Henney AM. The promise and challenge of personalized medicine: aging populations, complex diseases, and unmet medical need. Croat Med J 2012;53:207-10.

23. de Magalhães JP. The scientific quest for lasting youth: prospects for curing aging. Rejuvenation Res 2014;17:458-67.

24. Mohler J, Najafi B, Fain M, Ramos KS. Precision medicine: a wider definition. J Am Geriatr Soc 2015;63:1971-2.

25. Reitz C. Toward precision medicine in Alzheimer's disease. Ann Transl Med 2016;4:107.

26. Hampel H, O’Bryant SE, Castrillo JI, Ritchie C, Rojkova K, Broich K, Benda N, Nisticò R, Frank RA, Dubois B, Escott-Price V, Lista S. Precision medicine - the golden gate for detection, treatment and prevention of Alzheimer's disease. J Prev Alzheimers Dis 2016;3:243-59.

27. Zhu Y, Tchkonia T, Pirtskhalava T. The Achilles' heel of senescent cells: from transcriptome to senolytic drugs. Aging Cell 2015;14:644-58.

28. Roos CM, Zhang B, Palmer AK. Chronic senolytic treatment alleviates established vasomotor dysfunction in aged or atherosclerotic mice. Aging Cell 2016;15:973-7.

29. Zhu Y, Tchkonia T, Fuhrmann-Stroissnigg H. Identification of a novel senolytic agent, navitoclax, targeting the Bcl-2 family of antiapoptotic factors. Aging Cell 2016;15:428-35.

30. Rahnasto-Rilla M, Tyni J, Huovinen M, Jarho E, Kulikowicz T, Ravichandran S, A Bohr V, Ferrucci L, Lahtela-Kakkonen M, Moaddel R. Natural polyphenols as sirtuin 6 modulators. Sci Rep 2018;8:4163.

31. Kraig E, Linehan LA, Liang H, Romo TQ, Liu Q, Wu Y, Benavides AD, Curiel TJ, Javors MA, Musi N, Chiodo L, Koek W, Gelfond JAL, Kellogg DL Jr. A randomized control trial to establish the feasibility and safety of rapamycin treatment in an older human cohort: Immunological, physical performance, and cognitive effects. Exp Gerontol 2018;105:53-69.

32. Ratho L, Pant A, Awasthi H. An antidiabetic polyherbal phytomedicine confers stress resistance and extends lifespan in Caenorhabditis elegans. Biogerontology 2017;18:131-47.

33. Barzilai N, Crandall JP, Kritchevsky SB. Metformin as a tool to target aging. Cell Metab 2016;23:1060-5.

34. Strazhesko ID, Tkacheva ON, Akasheva DU. Atorvastatin therapy modulates telomerase activity in patients free of atherosclerotic cardiovascular diseases. Front Pharmacol 2016;7:347.

35. Kyriazis M. Translating laboratory anti-aging biotechnology into applied clinical practice: problems and obstacles. World J Transl Med $2015 ; 4: 51-4$. 
36. Joyner MJ. Precision medicine, cardiovascular disease and hunting elephants. Prog Cardiovasc Dis 2016;58:651-60.

37. MacRae CA, Vasan RS. Closing the phenotype gap in precision medicine. Circulation 2016;133:2634-9.

38. Bausch KC. The Theory and Practice of Third Phase Science. In: Metcalf G, editor. Social Systems and Design. Springer, Tokyo: Translational Systems Sciences; 2014.

39. Olshansky SJ. Is life extension today a faustian bargain? Front Med (Lausanne) 2017;4:215

40. Barat I, Andreasen F, Damsgaard EMS. Drug therapy in the elderly: what doctors believe and patients actually do. Br J Clin Pharmacol 2001;51:615-22.

41. Tang HY, Sayers SL, Weissinger G, Riegel B. The role of depression in medication adherence among heart failure patients. Clin Nurs Res 2014;23:231-44.

42. Donadiki EM, Jiménez-García R, Hernández-Barrera V. Health belief model applied to non-compliance with HPV vaccine among female university students. Public Health 2014;128:268-73.

43. Ehrlich S, Newkirk T, Pallas S, Riedinger T, Jackson D. Why are patients with chronic disease nonadherent with their prescription medications? Proceeding of American Academy of Physician Assistants 2017 conference; 2017 May 15-19; Las Vegas, USA.

44. Pasina L, Brucato AL, Falcone C, Cucchi E, Bresciani A, Sottocorno M, Taddei GC, Casati M, Franchi C, Djade CD, Nobili A. Medication non-adherence among elderly patients newly discharged and receiving polypharmacy. Drugs Aging 2014;31:283-9.

45. Mukhtar O, Weinman J, Jackson SH. Intentional non-adherence to medications by older adults. Drugs Aging 2014;31:149-57.

46. Shepherd JG, Locke E, Zhang Q. Health services use and prescription access among uninsured patients managing chronic diseases. J Community Health 2014;39:572-83.

47. Domingue BW, Belsky DW. The social genome: current findings and implications for the study of human genetics. PLoS Genet 2017;13:e1006615.

48. Cole SW. Human social genomics. PLoS Genet 2014;10:e1004601.

49. Portin P. A comparison of biological and cultural evolution. J Genet 2015;94:155-68.

50. Creanza N, Kolodny O, Feldman MW. Cultural evolutionary theory: how culture evolves and why it matters. Proc Natl Acad Sci U S A 2017; doi: 10.1073/pnas.1620732114.

51. Zierer J, Menni C, Kastenmüller G, Spector TD. Integration of 'omics' data in aging research: from biomarkers to systems biology. Aging Cell 2015;14:933-44.

52. Kyriazis M. Technological integration and hyper-connectivity: tools for promoting extreme human lifespans. Complexity 2014;20:15-24.

53. Kyriazis M. Reductionism versus Systems Thinking in Aging Research. In: Ahmad S. editor, Aging: Exploring a complex phenomenon. Boca Raton: CRC Press; 2018

54. Ogino S, Lochhead P, Chan AT. Molecular pathological epidemiology of epigenetics: emerging integrative science to analyze environment, host, and disease. Mod Pathol 2013;26:465-84.

55. Ogino S, Fuchs CS, Giovannucci E. How many molecular subtypes? Implications of the unique tumor principle in personalized medicine. Expert review of molecular diagnostics 2012;12:621-8.

56. Schully SD, Khoury MJ. What is translational genomics? An expanded research agenda for improving individual and population health. Appl Transl Genom 2014;3:82-3.

57. Heneghan C, Ben Goldacre B, Mahtani KR. Why clinical trial outcomes fail to translate into benefits for patients. Trials 2017;18:122.

58. Abernethy AP, Wheeler JL. True translational research: bridging the three phases of translation through data and behavior. Translational Behav Med 2011;1:26-30

59. London AJ, Kimmelman J. Why clinical translation cannot succeed without failure. eLife 2015;4:e12844.

60. Marusic U, Grosprêtre S. Non-physical approaches to counteract age-related functional deterioration: applications for rehabilitation and neural mechanisms. Eur J Sport Sci 2018;20:1-11.

61. Amendola LM, Robinson JO, Hart R, Biswas S, Lee K, Bernhardt BA, East K, Gilmore MJ, Kauffman TL, Lewis KL, Roche M, Scollon S, Wynn J, Blout C. Why patients decline genomic sequencing studies: experiences from the CSER consortium. J Genet Couns 2018; doi: 10.1007/s10897-018-0243-7.

62. Wolf SM, Burke W, Koenig BA. Mapping the ethics of translational genomics: situating return of results and navigating the research-clinical divide. J Law Med Ethics 2015;43:486-501. 